\title{
FES Proportional Tuning Based on sEMG
}

\author{
Yu Zhou ${ }^{1}$, Jia Zeng ${ }^{1}$, Kairu $\mathrm{Li}^{2}$, and Honghai $\mathrm{Liu}^{1,2}$ \\ ${ }^{1}$ State Key Laboratory of Mechanical System and Vibration, \\ Shanghai Jiao Tong University, Shanghai, China \\ $\{$ hnllyu, jia.zeng, honghai.liu\}@sjtu.edu.cn, \\ http://bbl.sjtu.edu.cn/ \\ ${ }^{2}$ Group of Intelligent System and Biomedical Robotics, \\ School of Computing, University of Portsmouth \\ Portsmouth, United Kingdom \\ \{kairu.li, honghai.liu\}@port.ac.uk
}

\begin{abstract}
It is evident that inappropriate functional electrical stimulation(FES) intensity is easy to trigger muscle fatigue and discomfortableness. This study proposes a FES tuning solution based on surface electromyography (sEMG), which is to form the relationship from sEMG to FES pulse width through the force. Six healthy subjects were invited to verify the proposed method based on the grip experiment. The feasibility of the estimated FES pulse width was evaluated respect to the correlation $\operatorname{index}(R)$ between the voluntary grip force and the FES-induced grip force. The experimental results indicated that the estimated pulse width could well induce the grip force that is similar to the voluntary force $(R>0.9)$, demonstrating the effectiveness of the proposed method and confirming the potential for improving the experience of FES in clinical settings.
\end{abstract}

Keywords: Functional electrical stimulation (FES), surface electromyography (sEMG), signal processing.

\section{Introduction}

FES has been an effective neuroprosthetic technique to restore lost motor function for spinal cord injury or stroke patients through delivering electrical stimulation current to their paralyzed muscles, since first used for foot drop rehabilitation by Liberson in 1960 [1,2]. However, there still lack of clear relationship between the paralyzed muscle physiological status and FES intensity, thus FES in clinical settings is usually passive and empirically tuned by the therapist. During FES-induced muscle contraction, the motor units are recruited in a synchronous and constant order manner, which consume more energy compared with voluntary muscle contraction [3]. So the empirically predefined stimulation patterns are easier to cause excessive stimulation that will speed up muscle fatigue, especially for the SCI patients whose fatigue resistance decreases in the weak muscle [4]. Besides, the passive FES excludes the active participation of the 
subjects and decreases the neuromuscular activity as well as energy consumption $[5,6]$. Thus, the relationship between the muscle status and FES intensity should be investigated to drive the closed-loop FES research forward and further improve the rehabilitation efficiency.

The muscle status can be reflected by muscle force, no matter for FESinduced muscle contraction or voluntary muscle contraction, thus we can use force as the bridge to combine muscle status and FES parameters. That is, if the relationship between muscle status and muscle force, muscle force and FES intensity are detected, then the relationship between muscle status and FES intensity can be obtained. The sEMG signal, demonstrating the physiological process of muscle contraction, is one of the dominant biological signals widely applied in human-machine interface [7]. Many studies focused on estimating muscle force information based on sEMG processing. For example, the nonnegative matrix factorization method was developed to estimate neural control information from sEMG to predict wrist force [8], the finger force with isometric contraction (all the fingers were held straight and in line with the metacarpals) was estimated based on the sEMG signals captured from forearm [9], and the FES-induced joint torque was estimated based on the evoked sEMG [10]. Thus, sEMG was applied in this study to recognise muscle status.

This study proposes an attempt of modeling the relationship between muscle status and FES parameters based on the grip movement. The Gaussian mixture regression (GMR) combined with polynomial fitting was applied as the solution of sEMG-to-FES modeling. The GMR is developed to map the sEMG-to-force relationship while the polynomial fitting represents the force-to-FES model. The findings in this study would pave the way for potential active closed-loop neuroprosthesis applications.

\section{Platform Construction}

The proposed method is a kind of muscle to muscle interface, as shown in Fig. 1. There are two main parts included in the frame, namely the sEMG/FES integration hardware and the FES parameter estimation algorithm. The customized integration hardware was designed in our previous study [11], which is used to acquire sEMG signals from the reference muscle and output the stimulation current to the target muscle. The raw sEMG signals are sampled at $1 \mathrm{kHz}$ and band-passe filtered $(20-500 \mathrm{~Hz})$ to remove the movement artifacts. After that, the digitized sEMG signals are transmitted to the host computer through bluetooth by the integration hardware for FES parameter estimation. The GMR is combined with polynomial fitting to form the FES parameters estimation algorithm. GMR is a probabilistic regression method which can be applied based on the parameters of a Gaussian mixture model (GMM) [12]. The pre-trained GMR model estimate muscle force $F_{p}$ based on the sEMG, then the estimated force is used as the input of the pre-trained polynomial fitting model to predict the FES parameters as defined by Eq.1 and Eq. 2. Finally, the estimated FES current is 
applied to the target muscle through the integration hardware to reproduce the similar muscle force as the reference muscle.

$$
\begin{gathered}
f(x)=p_{1} x^{n}+p_{2} x^{n-1}+\ldots+p_{n} x+p_{n+1} \\
\widehat{P}=f\left(F_{p}\right), \widehat{P} \in\left[H_{l}, H_{u}\right]
\end{gathered}
$$

where $f$ is the mathematical model for $n$ degrees polynomial fitting of FESinduced muscle force and FES parameters, and $\widehat{P}$ is the estimated FES parameters. The lower threshold $H_{l}$ was defined as the stimulation intensity that induced the muscle contraction slightly, while the upper threshold $H_{u}$ was the stimulation intensity that made the subjects feel uncomfortable.

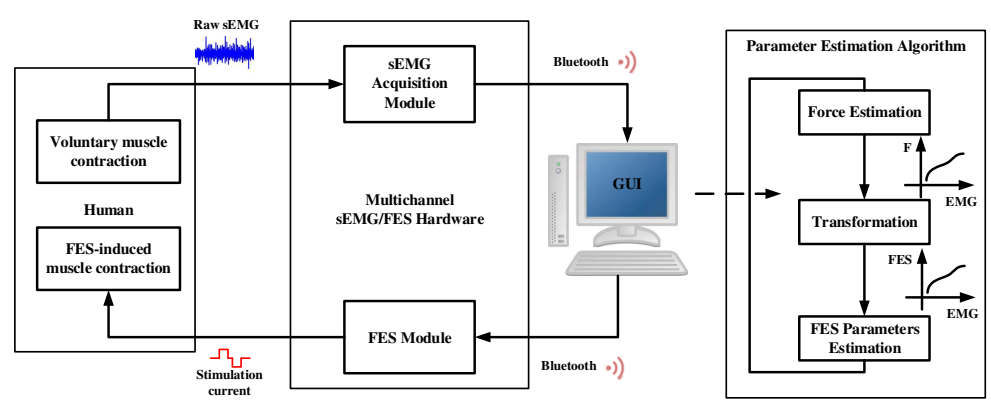

Fig. 1: The framework of the proposed sEMG to FES interface. The solid arrows represent the data/signal flow.

\section{Experimental Protocols}

\subsection{Subjects}

Six healthy subjects (aged 23 to 28; referenced as S1-S6) were invited to participate in the experiment. None of them had any history of neuromuscular disorder and each gave written informed consent prior to the experiment. Al1 the experiment procedures in this study were approved by the SJTU School Ethics Committee.

\subsection{Preliminary Experiment}

The grip force and the FES pulse width were selected in this study to verify the proposed method. The grip dynamometer (Biometrics Ltd, UK) was used to measure the grip force. And the Flexor Digitorum Superficialis muscle(FDS) was selected as the target muscle for sEMG acquisition and FES stimulation. 
Table 1: The FES intensity range for different subjects.

\begin{tabular}{ccc}
\hline \hline Subject & $\begin{array}{c}\text { Upper threshold } \\
\text { Pulse Width }(\mu \mathrm{s})\end{array}$ & $\begin{array}{c}\text { Lower threshold } \\
\text { Pulse Width }(\mu \mathrm{s})\end{array}$ \\
\hline S1 & 390 & 10 \\
S2 & 250 & 100 \\
S3 & 250 & 50 \\
S4 & 240 & 20 \\
S5 & 290 & 10 \\
S6 & 250 & 10 \\
\hline \hline
\end{tabular}

The preliminary experiment setup is shown in Fig. 2(a). The purpose of the preliminary experiment was to find the upper and lower stimulation threshold in Eq.2 for each subject in terms of the pulse width. The results are shown in Table 1 . The pulse width of the stimulation current was gradually increased from the lower threshold to the upper threshold with a constant increment and applied to the FDS muscle of each subject with the grip force measured. During the preliminary experiment, the stimulation frequency was fixed as $50 \mathrm{~Hz}$ and the current amplitude was fixed as the medium value of the lower and upper amplitude threshold of the subject when the pulse width was $100 \mu \mathrm{s}$.

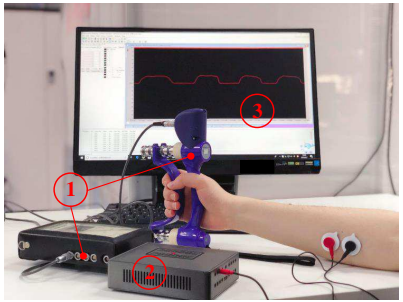

(a)

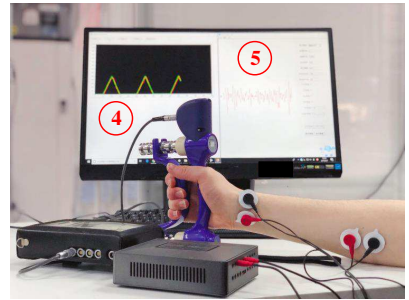

(b)

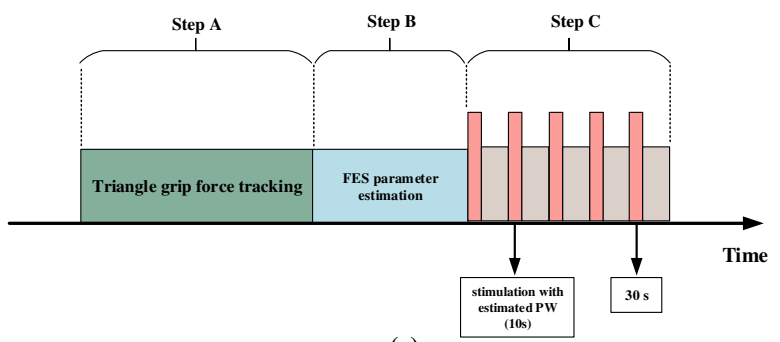

(c)

Fig. 2: (a) The preliminary experiment; (b) The formal experiment; (c) The experimental procedures; 1 . The grip dynamometer; 2 . The multichannel sEMG/FES hardware; 3 . The grip force induced by FES; 4. The triangular grip force; 5 . The recorded sEMG signals. 


\subsection{Formal Experiment}

The formal experiment setup is shown in Fig. 2(b) and the experimental flow is shown in Fig. 2(c). The two differential sEMG electrodes were placed on the FDS muscle belly in the right hand, parallel to the direction of muscle fiber, with the reference electrodes placed on the end of the ulna. For the step A, the subjects sat comfortably in front of a computer screen and lay his right elbow on the desk. The subjects performed right hand grip and control the grip force to track the triangular wave force line displayed in the computer screen for $60 \mathrm{~s}$, with sEMG from FDS and grip force recorded simultaneously. The recorded 60 $\mathrm{s}$ sEMG data and force data were used to estimate the FES pulse width by the proposed method during step B. After the pulse width estimation, the current with the estimated pulse width was applied to FDS muscle belly through the pair of sEMG electrodes (the frequency and the amplitude of the current were fixed as the values in the preliminary experiment) in step C. The stimulation was repeated for five times and each stimulation lasted for 10s with a 30s rest followed to avoid muscle fatigue. The FES-induced grip force of the right hand was recorded during the stimulation period of each subject.

\section{Data Processing and Evaluation Metrics}

The sEMG signals were segmented by the sliding data window with a length of 300 data points and the step size was set as 50 data points. The time domain (TD)features [13], root-mean-square values (RMS) [14] and sixth order autoregressive coefficient (AR6) [15] were calculated from each sliding data window to make up the feature vector. The feature vector was combined with the corresponding grip force to form the data sets for GMR model training and grip force estimation. The offline force estimation accuracy was determined using a five-fold estimation cross-validation.

1) Optimal polynomial fitting: The optimal polynomial fitting model $f$ in Eq.1 that representing the relationship between the FES-induced grip force and FES current pulse width was investigated. The polynomial fitting was compared from one degree to three degrees respect to the coefficient of determination $\left(R^{2}\right)$. The one-way ANOVA was applied to investigate the fitting performance between different degrees. The level of statistical significance was set at $p<0.05$ for all statistics.

2) Grip force estimation accuracy: Since the grip force is used as a bridge to link sEMG and FES parameter, the force estimation accuracy is related to FES current pulse width estimation. The coefficient of determination $\left(R^{2}\right)$ and the normalized root-mean-square error (NRMSE) between the estimated values and the real values are used to quantize the estimation accuracy. And a oneway ANOVA was applied to compare the effects of different feature sets on the estimation accuracy.

3) Correlation between voluntary and FES - induced force: This index is used to verify the effectiveness of the estimated FES current in retrieving 
force with FES-induced muscle contraction. The FES pulse width is estimated based on the sEMG signals that respect to voluntary force in step A in formal experiment. The normalized FES-induced force is compared with the normalized original voluntary force in terms of the Pearsons correlation coefficient $(R)$.

\section{$5 \quad$ Results}

\subsection{Optimal Polynomial Fitting}

The recorded force induced by the stimulation current with different pulse width was calculated as the average values of force during stimulation as shown in Fig. $3(\mathrm{a})$, then the different pulse width was polynomial fitted with the corresponding FES induced force as shown in Fig. 3(b). The average goodness of fit across all the subjects are shown in Fig. 3(c) based on the data captured in the preliminary experiment, and the three degrees polynomial fitting shows the best performance $\left(R^{2}=0.91 \pm 0.05\right)$. No significant differences appealed between the effects of different fitting degrees $(p>0.05)$. The two degrees polynomial fitting was selected to set up the mathematical model $f$ for the rest of this study, considering the trade-off between computing complexity and goodness of fit.
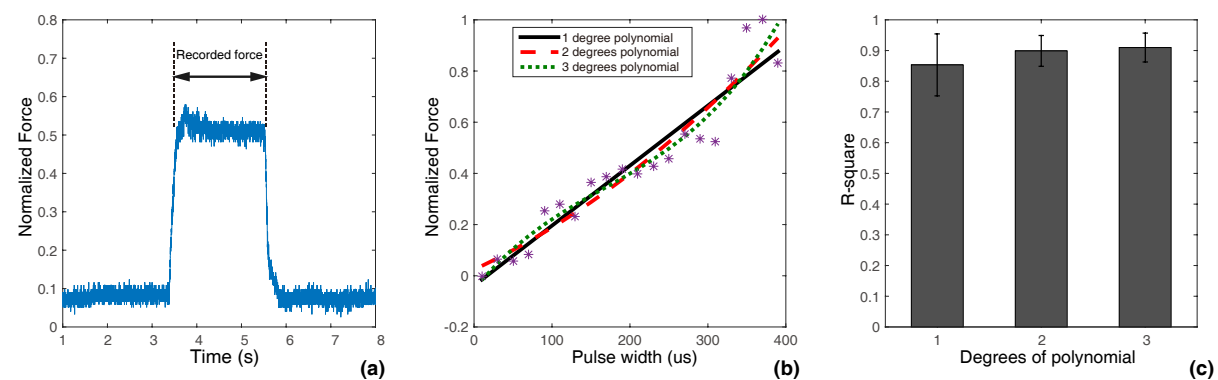

Fig. 3: (a) Demonstration for grip force calculation during FES. (b) Demonstration for polynomial fitting process. (c) The average results for different degrees of polynomial fitting performance across all the subjects.

\subsection{Grip Force Estimation Accuracy}

The grip force estimation results are shown in Fig. 4. The average results across all the subjects revealed that GMR showed excellent performance for decoding grip force based on the RMS feature sets $(R=0.950 \pm 0.023, N R M S E=6.15 \% \pm$ $1.31 \%)$ and TD feature sets $(R=0.954 \pm 0.018, N R M S E=5.96 \% \pm 1.09 \%)$. The ANOVA analysis indicated that no significant differences were found between the performance of RMS and TD feature sets $\left(p=0.954\right.$ for $R^{2}$ and $p=0.919$ for NRMSE). Significant differences appeared between the RMS and AR6 feature 

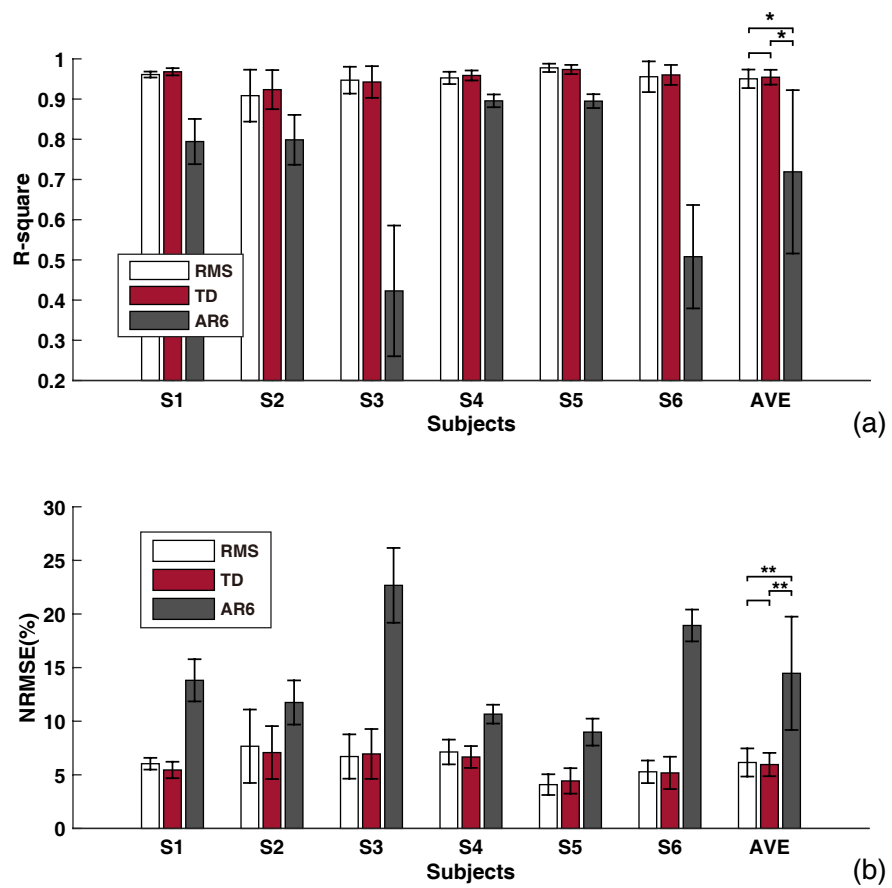

Fig. 4: The comparison of force estimation accuracy across the six subjects based on different sEMG features: (a) $R^{2}$; (b) NRMSE. Error bars represent the standard deviation. One asterisk "*" denotes $p<0.05$ and two asterisks "**" denote $p<0.001$.

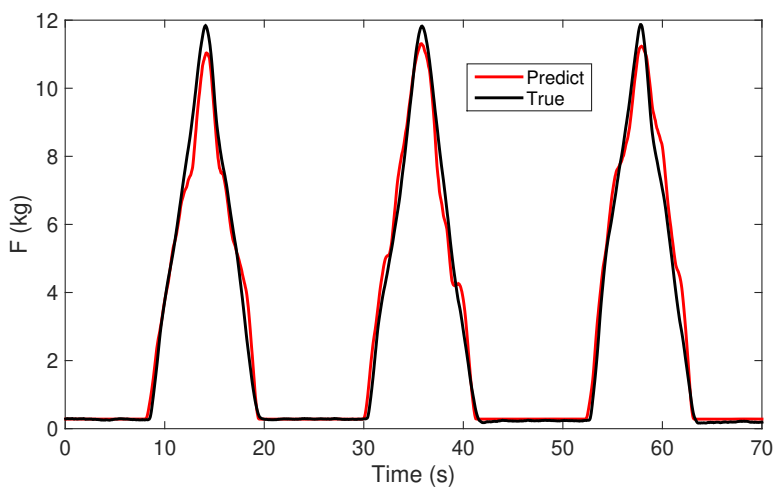

Fig. 5: Typical demonstrations of comparisons between true and predicted grip force for subject S1. 
sets $\left(p=0.004\right.$ for $R^{2}$ and $p<0.001$ for $N R M S E$ ) as well as between the TD feature sets and AR6 feature sets $\left(p=0.004\right.$ for $R^{2}$ and $p<0.001$ for NRMSE). Thus, the results through the rest of this study were based on the RMS feature sets considering the computing consumption. Fig. 5 shows an example of the estimation quality vividly based on the data sets of S1.

\subsection{Correlation Between Voluntary and FES-induced Force}

Table 2 shows the results of the similarity between the voluntary force and corresponding FES-induced force across all the subjects. The FES-induced force is highly correlated with the voluntary force $(R>0.9)$ except for S2 and the mean value across all subjects is $R=0.93 \pm 0.08$, demonstrating that the FES estimated based on voluntary sEMG is effective to reproduce the grip force as similar as the voluntary force. Fig. 6 shows an example of the differences between the estimated-FES-induced grip force and voluntary grip force.

Table 2: The Results for correlation between voluntary and FES-induced force.

\begin{tabular}{ccccccccc}
\hline \hline Subject & $\mathrm{S} 1$ & $\mathrm{~S} 2$ & $\mathrm{~S} 3$ & $\mathrm{~S} 4$ & $\mathrm{~S} 5$ & $\mathrm{~S} 6$ & $\mathbf{M E A N} \pm \mathbf{S d}$ \\
\hline $\mathrm{R}$ & 0.96 & 0.79 & 0.98 & 0.97 & 0.97 & 0.90 & $\mathbf{0 . 9 3} \pm \mathbf{0 . 0 8}$ \\
\hline \hline
\end{tabular}
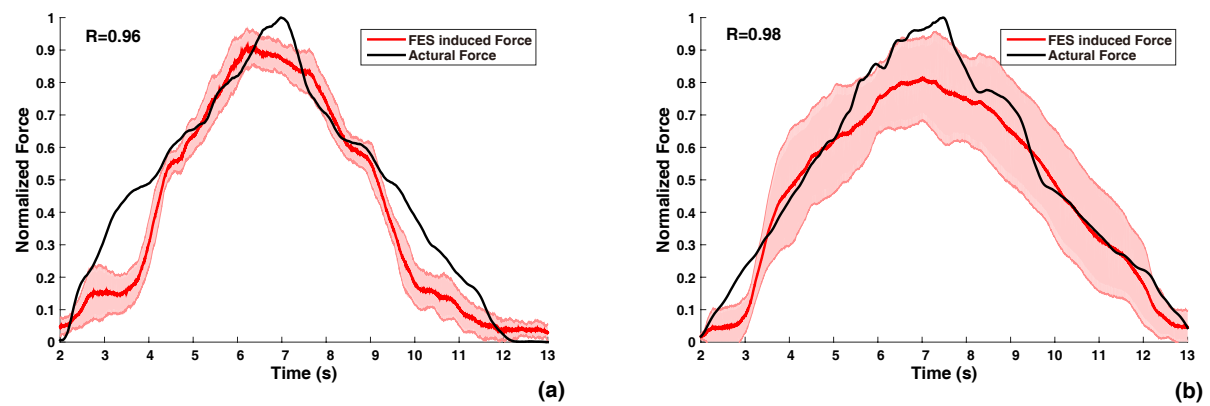

Fig. 6: Demonstrations of comparisons between voluntary force and FES-induced force based on the experimental data of S1 and S3: (a) S1, (b) S3. The light red region represents the standard deviation of the five repetitions of FES-induced grip force. The red solid line represents the average values of the five repetitions of FES-induced grip force. 


\section{Discussion and Conclusion}

This study investigated the sEMG-to-FES sequential mapping relationship based on the proposed method, which combined GMR with polynomial fitting. GMR is used to model the sEMG-to-force relationship while polynomial fitting is applied to model the force-to-FES relationship. The experimental results verified the feasibility of the proposed muscle-to-muscle interface, which is potential for the force training applications based on the closed-loop FES.

The relationship between FES current pulse width and the correlated FESinduced grip force was investigated through polynomial fitting. As shown in Fig. $3(\mathrm{c})$, the three degrees polynomial fitting shows the best performance compared with the other two kinds of fitting, indicating the non-linear relationship between the FES pulse width and FES-induced grip force. The grip force is applied as the bridge for modeling the sEMG-to-FES relationship. Thus the force estimation accuracy is vital for the sEMG-to-FES estimation. The GMR model was developed to predict grip force based on the voluntary sEMG signals captured form FDS muscle. As shown in Fig. 4 and Fig. 5, the performance of GMR was regard as excellent on proportional force prediction, based on RMS feature and TD feature. Thus, the sEMG-to-force relationship and the force-to-FES relationship were setup to ensure the accuracy of sEMG-to-FES estimation.

The FES pulse width was estimated by the proposed method based on the voluntary sEMG, then the voluntary grip force corresponding to the aforementioned voluntary sEMG was compared with the grip force induced by the FES current with estimated pulse width. The results in Table 2 and Fig. 6 indicated the high similarity of the force pattern between the voluntary muscle contraction and estimated-FES-induced muscle contraction. Thus, it revealed that the proposed method could well stimulate the target muscle to generate the desired grip force according to the voluntary grip movement intention.

As a muscle-to-muscle interface, the proposed method could be used for the closed-loop FES applications. The FES parameters can be estimated based on the target muscle contraction and be applied to the same muscle to help strengthen the muscle force. Besides, the FES intensity is adapt to muscle status, which could avoid excessive stimulation and slow down the muscle fatigue compared with the open loop FES. To drive the method to be practical for clinical applications, the random grip force will be involved in the near future. Besides, more efforts will be devoted to drive the proposed method to stroke rehabilitation, that is, the FES parameters will be estimated based on the sEMG and force captured from the reference unaffected hand muscle isometric contraction and be evaluated on the contralateral muscle in the affected hand to see wether the estimated-FES-induced contralateral muscle force is similar to the reference muscle.

Acknowledgments. This work is supported by the National Natural Science Foundation of China (No. 51575338, 51575407, 51475427,61733011) and the Fundamental Research Funds for the Central Universities (17JCYB03). 


\section{References}

1. Lynch, C.L., Popovic, M.R.: Functional electrical stimulation. IEEE control systems 28(2), 40-50 (2008)

2. Liberson, W.: Functional electrotherapy: stimulation of the peroneal nerve synchronized with the swing phase of the gait of hemiplegic patients. Arch Phys Med Rehabil 42, 101 (1961)

3. Binder-Macleod, S.A., Snyder-Mackler, L.: Muscle fatigue: clinical implications for fatigue assessment and neuromuscular electrical stimulation. Physical therapy 73(12), 902-910 (1993)

4. Pelletier, C., Hicks, A.: Muscle fatigue characteristics in paralyzed muscle after spinal cord injury. Spinal Cord 49(1), 125 (2011)

5. Edgerton, V.R., Roy, R.R.: Robotic training and spinal cord plasticity. Brain research bulletin 78(1), 4-12 (2009)

6. Lotze, M., Braun, C., Birbaumer, N., Anders, S., Cohen, L.G.: Motor learning elicited by voluntary drive. Brain 126(4), 866-872 (2003)

7. Zhou, Y., Liu, J., Zeng, J., Li, K., Liu, H.: Bio-signal based elbow angle and torque simultaneous prediction during isokinetic contraction. Science China Technological Sciences 62(1), 21-30 (2019)

8. Jiang, N., Englehart, K.B., Parker, P.A.: Extracting simultaneous and proportional neural control information for multiple-dof prostheses from the surface electromyographic signal. IEEE Transactions on Biomedical Engineering 56(4), 1070-1080 (2009)

9. Castellini, C., Koiva, R.: Using surface electromyography to predict single finger forces. In: 2012 4th IEEE RAS \& EMBS International Conference on Biomedical Robotics and Biomechatronics (BioRob). pp. 1266-1272. IEEE (2012)

10. Li, Z., Guiraud, D., Andreu, D., Benoussaad, M., Fattal, C., Hayashibe, M.: Realtime estimation of fes-induced joint torque with evoked emg. Journal of neuroengineering and rehabilitation 13(1), 60 (2016)

11. Zhou, Y., Fang, Y., Gui, K., Li, K., Zhang, D., Liu, H.: semg bias-driven functional electrical stimulation system for upper-limb stroke rehabilitation. IEEE Sensors Journal 18(16), 6812-6821 (2018)

12. Calinon, S., Guenter, F., Billard, A.: On learning, representing, and generalizing a task in a humanoid robot. IEEE Transactions on Systems, Man, and Cybernetics, Part B (Cybernetics) 37(2), 286-298 (2007)

13. Hudgins, B., Parker, P., Scott, R.N.: A new strategy for multifunction myoelectric control. IEEE Transactions on Biomedical Engineering 40(1), 82-94 (1993)

14. Huang, Y., Englehart, K.B., Hudgins, B., Chan, A.D.: A gaussian mixture model based classification scheme for myoelectric control of powered upper limb prostheses. IEEE Transactions on Biomedical Engineering 52(11), 1801-1811 (2005)

15. Khezri, M., Jahed, M.: A neuro-fuzzy inference system for semg-based identification of hand motion commands. IEEE Transactions on Industrial Electronics 58(5), 1952-1960 (2011) 